\title{
A two-step initial mass function:
}

\section{Consequences of clustered star formation for binary properties}

\author{
R. H. Durisen ${ }^{1,2}$, M. F. Sterzik ${ }^{3}$, and B. K. Pickett ${ }^{4,5}$ \\ 1 Max-Planck-Institut für extraterrestrische Physik, 85740 Garching bei München, Germany \\ 2 Department of Astronomy, SW319, Indiana University, Bloomington, Indiana 47405, USA \\ 3 European Southern Observatory, Alonso de Cordova 3107, Vitacura, Casilla 19001, Santiago 19, Chile \\ 4 Department of Physics and Astronomy, Neils Science Center, Valparaiso University, Valparaiso, Indiana 46383, \\ USA \\ 5 Department of Chemistry and Physics, Purdue University Calumet, 2200 169th Street, Hammond, \\ Indiana 46323, USA
}

Received 4 September 2000 / Accepted 9 March 2001

\begin{abstract}
If stars originate in transient bound clusters of moderate size, these clusters will decay due to dynamic interactions in which a hard binary forms and ejects most or all the other stars. When the cluster members are chosen at random from a reasonable initial mass function (IMF), the resulting binary characteristics do not match current observations. We find a significant improvement in the trends of binary properties from this scenario when an additional constraint is taken into account, namely that there is a distribution of total cluster masses set by the masses of the cloud cores from which the clusters form. Two distinct steps then determine final stellar masses - the choice of a cluster mass and the formation of the individual stars. We refer to this as a "two-step" IMF. Simple statistical arguments are used in this paper to show that a two-step IMF, combined with typical results from dynamic few-body system decay, tends to give better agreement between computed binary characteristics and observations than a one-step mass selection process.
\end{abstract}

Key words. methods: statistical - stars: binaries: general - stars: formation - stars: pre-main-sequence

\section{Introduction}

\subsection{Two-step initial mass function}

One of the fundamental goals of star formation theory is to determine the relationship between molecular cloud conditions and the mass and multiplicity distributions of the resulting stars. To address aspects of this problem, Sterzik \& Durisen (1995, 1998, hereafter SD95 and SD98, respectively) used the Mikkola \& Aarseth (1990, 1993) chain regularizaton code to compute the dynamic decay of nonhierarchical $N$-body clusters with $N=3,4$, and 5 . It was already known from classic work of the 1970's and earlier that, in the absence of dissipation, the decay of pure point-mass few-body systems is dominated, within a few tens of system crossing times, by the formation of a hard binary which ejects most if not all the other stars (e.g., van Albada 1968a,b; Standish 1972; Saslaw et al. 1974; Harrington 1974, 1975; Heggie 1975; Monaghan 1976a,b; Valtonen 1976). In SD95 and SD98, the few-body systems were explicitly interpreted as products of molecular cloud core collapse and fragmentation. The decay products were

Send offprint requests to: R. H. Durisen, e-mail: durisen@astro.indiana.edu characterized statistically by considering large numbers of cluster calculations for each choice of $N$ with the goal of determining distribution functions of fragment properties, such as binary fractions and mass-ratio distributions.

For statistical studies of few-body systems, one must adopt a procedure for choosing component stellar masses. One common approach (e.g., McDonald \& Clarke 1993, hereafter McDC93) is to pick masses at random from an assumed stellar mass spectrum (SMS). In this case, the SMS is also the overall IMF for the stars. SD98 also introduced a two-step process where they first chose a cluster total mass from a cluster mass spectrum (CMS) and then required that the sum of the stellar masses, chosen randomly from a different stellar mass spectrum (SMS), should equal the chosen cluster mass. In this approach, because random mass selections from the SMS which do not match the chosen cluster mass are discarded, the final IMF of the selected stars differs from the SMS through the imposition of the total cluster mass constraint. In this paper, we refer to the distribution of stellar masses which results from a large ensemble of such two-step choices as a "twostep" IMF. SD98 found that the distributions of some binary properties agree better with observations when stellar masses are chosen in this way. The distribution which 
improves the most is the overall binary fraction $B F$ as a function of stellar mass $M$.

The reason for the improvement is straightforward. Few-body cluster decay most often leads to production of one hard binary from the two most massive stars, an effect referred to as "dynamical biasing". Assuming this always occurs, i.e., "complete" dynamical biasing, McDC93 demonstrated that, when stars are chosen at random from a realisitic IMF in a one-step process, solar-mass stars almost always end up being in binaries for small $N$. On the other hand, observations show that the $B F$ for nearby solar-type stars in the field is only $0.57 \pm 0.09$ (Duquennoy \& Mayor 1991, hereafter DM91). A BF this low can be attained for $N \geq 10$ or so, but then the mass ratio distribution does not agree well with observations. Moreover, for $N \geq 10$, the $B F$ for $\mathrm{M}$ stars becomes too low. Observations of field $\mathrm{M}$ stars suggest $B F$ in the range $0.26 \pm 0.09$ (Leinert et al. 1997, hereafter L97) to $0.42 \pm 0.09$ (Fischer \& Marcy 1992, hereafter FM92). With a two-step IMF, when total cluster masses are chosen from a moderately steep and broad CMS, solar-mass stars are often not the most massive stars in their clusters. By the same token, for the same $N, \mathrm{M}$ stars more often are the most massive stars. The overall result is a more uniform distribution of $B F$ with mass from a single value of $N$.

\subsection{Motivations and goals}

A two-step IMF might be a reasonable representation of star cluster formation because the masses of stars in a small cluster must be constrained to some extent by the total cloud mass from which the cluster forms. For many years, Larson (1992) has advocated that processes on different physical scales imprint themselves on the IMF; and Elmegreen (1997) has also proposed a related two-process IMF where the low and high-mass parts of the IMF are shaped by different physical constraints. Observations by Motte et al. (1998, hereafter Mot98) of $\rho$ Ophiuchus indicate that pre-collapse cloud cores can be already "fragmented" into few-body systems of stellar mass clumps. These clumps do appear to have the same mass spectrum as the IMF of young stars in the same region (Luhman \& Rieke 1999; Testi \& Sargent 1998). The observations seem consistent with the "prompt fragmentation" scenario (Clarke \& Pringle 1991, 1993) which is the starting point of the McDC93 analyses.

Of course, there are other ways to explain the observed $B F(M)$. For instance, McDonald \& Clarke (1995, hereafter McDC95) showed that cluster evolutions which include the dissipative effects of collisions involving massive circumstellar disks give a flatter $B F(M)$. Cluster evolution will also deviate from point-mass dynamics when cluster fragments accrete competitively in a gas-rich environment (e.g., Zinnecker 1982; Burkert \& Bodenheimer 1996; Bonnell et al. 1997; 1998, Smith et al. 1997; Bate 1999). One could also appeal to slow dissolution of clus-
Table 1. Fractions of different binary pairings for the SD98 two-step IMF

\begin{tabular}{lccc}
\hline$N$ & $M_{1} M_{2}$ & $M_{1} M_{3}$ & $M_{2} M_{3}$ \\
\hline 3 & 0.80 & 0.19 & 0.01 \\
4 & 0.81 & 0.15 & 0.03 \\
5 & 0.81 & 0.15 & 0.04 \\
$\mathrm{MC}$ & 0.82 & 0.15 & 0.03 \\
\hline
\end{tabular}

ters through loss of residual gas. All these effects would drive results away from pure dynamical biasing.

In light of the many uncertainties, we do not claim that a two-step IMF is the final or only answer. Our objective here is to illustrate, by using reasonable choices of the CMS and SMS and by making some simple statistical calculations based on the results of SD98, that a two-step IMF does improve binary characteristics and therefore needs to be kept in mind as a possibly important component of the star formation process, at least in a statistical sense. We do not attempt to explain the stellar IMF itself but choose our parameters so that the IMF resulting from our Monte Carlo approach is reasonable.

Section 2 describes our methodology and offers some motivation for our choices of mass spectra and other parameters. Our main results are given in Sect. 3 and discussed briefly in Sect. 4. Section 5 summarizes our conclusions.

\section{Methodology}

\subsection{Deviations from complete dynamical baising}

SD98 found that complete dynamical biasing is violated in two ways. First, as $N$ increases, decay channels which involve stable and metastable hierarchical multiples or which yield two binaries become more common. Even so, only one stellar pair generally has the bulk of the original cluster binding energy; and, far and away, the most common decay channels are single stars plus one binary or one multiple fragment. For example, such decays occurred for $>93 \%$ of the 1000 two-step IMF $N=5$ clusters considered in SD98. The second deviation from complete dynamical biasing is that resultant binaries do not always consist of the two most massive stars. Table 1 , which is based on data from SD98, shows the fraction of binary decay fragments with different mass pairings for the two-step IMF from that paper. Here $M_{1}, M_{2}$, and $M_{3}$ are the first, second, and third-most massive stars, respectively. Pairings of lower mass components are negligible $(<1 \%)$. The last row of Table 1 is explained below.

\subsection{Approximate Monte Carlo approach}

Integrations like those of SD98 are somewhat computationally intensive, which limits ensembles of integrations to about 1000 systems. Nevertheless, properties of the 
results described in the above section are fairly robust. In order to consider a wide range of parameter space with much larger numbers of systems for each choice of parameters, we here adopt a simple Monte Carlo (MC) approach, similar to that of McDC93, but modified by incorporation of a two-step IMF and the deviations from complete dynamical biasing given in Table 1 .

We first choose a CMS $f_{\mathrm{c}}\left(M_{\text {tot }}\right)$ and a SMS $f_{\mathrm{s}}(M)$, including functional forms and upper and lower mass limits. An ensemble of cluster masses is then generated randomly from $f_{\mathrm{c}}$. For each $M_{\text {tot }}$, stellar masses $M_{i}$ are chosen from $f_{\mathrm{s}}$ until $M_{\text {tot }}$ is matched within some prescribed tolerance $\tau$ according to

$(1-\tau) M_{\mathrm{tot}} \leq \sum_{i=1}^{N} M_{i} \leq(1+\tau) M_{\mathrm{tot}}$.

We pick the stellar masses in two alternative ways: A) Fixed $N . N$ stellar masses are chosen repeatedly at random from the adopted $f_{\mathrm{s}}$ until Eq. (1) is satisfied. B) Variable $N$. The test in Eq. (1) is made after each stellar mass is chosen. If Eq. (1) is satisfied at any point in the process, then this determines the cluster size $N$ as well as the stellar masses. We impose an upper limit $N_{\max }$ on the allowed $N$. If (1) is not satisfied by the time $N=N_{\max }$, a new selection of stellar masses is begun starting with one star.

Suppose we now have an ensemble of clusters (typically 100000 for a given choice of $f_{\mathrm{c}}, f_{\mathrm{s}}$, and $N$ or $\left.N_{\max }\right)$. We assume, as in McDC93, that each cluster decays into one binary plus single stars. In light of the SD98 results, this is consistent with two interpretations: 1) most multiples resulting from the dominant decay channels (singles plus one multiple) do eventually decay into one hard binary plus singles. 2) Even though some multiples may be stable and not decay, the hardest binaries in the multiples are effectively included in the $B F$ statistics, with only small errors introduced by treating the extra members of multiples as if they were single stars. For field stars, the DM91 $B F=0.57 \pm 0.09$ does in fact include a contribution from triples and quadruples at about the same level as the stated uncertainty in $B F$. In young stellar populations, multiples may be more common and have a greater effect on the statistics (see Zinnecker \& Mathieu 2001 and the references therein).

For each cluster, we arrange the masses $\left\{M_{i}\right\}$ so that they decrease monotonically as $i$ increases from 1 to $N$. When forming the binary, we assign probabilities to the various mass pairings according to the MC entries in Table 1. Statistics on the binary fraction as a function of mass $B F(M)$ for different choices of primary mass can then be compiled. The resultant two-step IMF $f_{i}(M)$ determined from all the stars in the clusters will be different from $f_{\mathrm{s}}$, because of the additional constraint imposed by $f_{\mathrm{c}}\left(M_{\text {tot }}\right)$ through Eq. (1).

For $N=4$ and 5, Table 2 compares the SD98 two-step IMF cluster integrations with the Monte Carlo procedure. The mass bins are the same as those used in SD98. Masses for this and all other tables are given in solar units. The MC results are based on 100000 fixed- $N$ clusters using the same CMS and SMS as in SD98. In Table 2, $B F^{+}$ represents $B F+T F+Q F$ where $T F$ and $Q F$ are the triple and quadruple fractions obtained in SD98. This is the proper quantity to compare with $B F(\mathrm{MC})$ because the $\mathrm{MC}$ approach assumes that multiples are effectively included in $B F$ through either of the interpretations given above. The fractions in these tables are computed such that the star in the given mass range is the primary. The MC numbers have statistical errors of less than a percent, while the SD98 numbers are uncertain by a few percent.

Table 2. Comparison of SD98 binary fractions with the Monte Carlo (MC) approach

\begin{tabular}{ccccc}
\hline$N$ & mass bin & $B F(\mathrm{SD} 98)$ & $B F^{+}(\mathrm{SD} 98)$ & $B F(\mathrm{MC})$ \\
\hline 4 & $0.1-0.2$ & 0.00 & 0.00 & 0.00 \\
& $0.2-0.5$ & 0.15 & 0.19 & 0.19 \\
& $0.5-1.2$ & 0.52 & 0.68 & 0.64 \\
& $>1.2$ & 0.73 & 0.93 & 0.89 \\
\hline 5 & $0.1-0.2$ & 0.01 & 0.01 & 0.00 \\
& $0.2-0.5$ & 0.15 & 0.25 & 0.21 \\
& $0.5-1.2$ & 0.35 & 0.60 & 0.55 \\
& $>1.2$ & 0.56 & 0.91 & 0.86 \\
\hline
\end{tabular}

The $B F(\mathrm{MC})$ 's in Table 2 tend to be systematically low by about 0.04 to 0.05 . This is due in part to the fact that the triples and quadruples lumped into $B F^{+}($SD98) can have more than one star in the same mass bin. Effectively, the most massive star in the multiple is counted as a binary primary in $\mathrm{BF}^{+}$. For comparison with $B F(\mathrm{MC})$, some stars from the same mass bin in the same multiple should be counted as singles but are not in the $B F^{+}$accounting. Also, when the multiples decay, the resulting binaries will not always contain the most massive star. Both these effects tend to inflate $\mathrm{BF}^{+}(\mathrm{SD} 98)$ relative to $B F(\mathrm{MC})$, but the differences are typically not much larger than the statistical uncertainties in the SD98 numbers.

Table 2 demonstrates that the MC approach does a reasonably good job of reproducing SD98 binary statistics, but the IMF resulting from the CMS and SMS chosen in SD98 is not realistic. Armed with the MC method, we can now consider binary statistics for other, more realistic mass distributions and for other ways of choosing cluster sizes without resorting to $N$-body calculations. One uncertainty is that we extrapolate SD98 results from $N \leq 5$ to $N>5$. $N$-body calculations of cluster decay for large ensembles of systems have not yet been done with $N>5$. We plan to remedy this deficiency in the near future. Meanwhile, Table 1 is an improvement over McDC93; and, anyhow, we find that our results are more affected by the two-step process than by modifications to dynamical biasing. 


\subsection{Choices for $f_{\mathrm{c}}\left(M_{\text {tot }}\right)$ and $f_{\mathrm{s}}(M)$}

Unfortunately, the physical processes which might underly the CMS and SMS are still poorly known. Because our goal in this paper is to illustrate the differences in binary characteristics between one-step and two-step approaches, we deliberately select our CMS and SMS so that, after two-step mass selection, they yield an acceptable stellar IMF.

\section{Our CMS is}

$$
f_{\mathrm{c}}\left(M_{\mathrm{tot}}\right) \sim M_{\mathrm{tot}}-\alpha_{\mathrm{c}}
$$

for $0.5 M_{\odot} \leq M_{\text {tot }} \leq 10.0 M_{\odot}$ and zero otherwise. Our standard choice for $\alpha_{\mathrm{c}}$ is 1.5. For the SMS, we use a lognormal distribution (see Scalo 1986)

$$
f_{\mathrm{S}}(M) \sim M^{-1} \exp \left\{-\left[\log _{10}\left(M / M_{\odot}\right)-\mu\right]^{2} / 2 \sigma^{2}\right\}
$$

for $M_{\min } \leq M \leq 10.0 M_{\odot}$ and zero otherwise with $\mu=$ $\log _{10} 0.20$ and $\sigma=\log _{10} 0.35$. The functions $f_{\mathrm{c}}$ and $f_{\mathrm{s}}$ give fractions of systems or stars per unit mass interval. Stars form from clouds with limited efficiency, however, and so the mass range of the CMS is lower than that of cloud cores. Mot98 note, for instance, that the pre-stellar clumps within the cores they observe represent only about $5 \%$ to $40 \%$ of the core gas mass, consistent with other estimates of star formation efficiency between $10 \%$ and $20 \%$ (Lada et al. 1991). So the cluster mass $M_{\text {tot }}$ represents some typical fraction of a molecular cloud core mass.

Parameters $\mu$ and $\sigma$ in the lognormal $f_{\mathrm{s}}$ were varied until $f_{i}$ from an $N_{\max }=10$ cluster ensemble agreed to within about $20 \%$ with the Kroupa et al. (1993, hereafter KTG93) piecewise power-law IMF over the range $0.3 M_{\odot}<M<3 M_{\odot}$ for $M_{\min }=0.08 M_{\odot}$. The KTG93 IMF is consistent with modern IMF's for stellar masses (Kroupa 1998) and is simple to characterize. It is a continuously decreasing mass spectrum with a powerlaw index of 1.3 for $0.08 M_{\odot}<M<0.5 M_{\odot}, 2.2$ for $0.5 M_{\odot}<M<1.0 M_{\odot}$, and 2.7 for $M>1.0 M_{\odot}$. Figure 1 compares the KTG93 IMF, $f_{\mathrm{s}}$, and $f_{i}$ for all stars in $100000 N_{\max }=10$ clusters created by the two-step IMF process with $\tau=0.1$. There is an excess of high-mass stars in $f_{i}$ relative to $f_{\mathrm{s}}$, because, for large cluster masses, random mass selections from $f_{\mathrm{s}}$ tend to be rejected until they include at least one high-mass star.

Although Scalo (1998) has recently disavowed the Miller \& Scalo (1979) lognormal IMF, others have argued for its plausibility (Adams \& Fatuzzo 1996). In Eq. (3), we use a lognormal for the SMS; but, after two-step mass selection, the two-step IMF is not lognormal. As shown in Fig. 1, it rolls over to a lognormal at low masses but has a power-law shape from about 0.6 to $6 M_{\odot}$. The CMS in (2) with $\alpha_{\mathrm{c}}=1.5$ has a power-law index similar to that of the molecular cloud mass spectrum (Elmegreen \& Falgarone 1996). Klessen \& Burkert (2000) might argue that (2) should be lognormal as well. We will offer some discussion of how parameter and function choices affect results in Sects. 3.5 and 3.6.

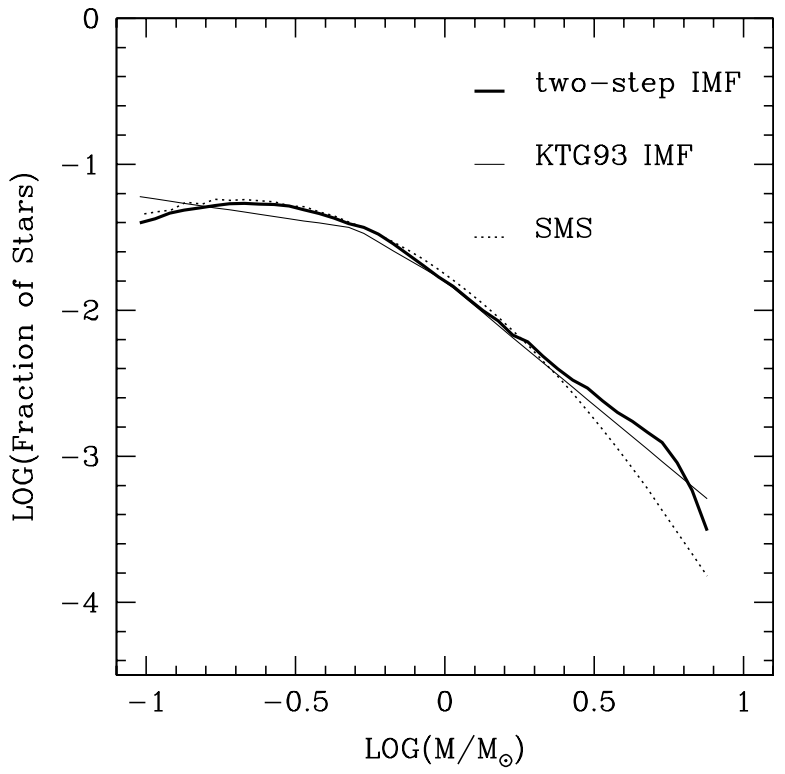

Fig. 1. The SMS $f_{\mathrm{s}}$ (dotted) and resultant two-step IMF $f_{i}$ (thick solid curve) for the $N_{\max }=10$ ensemble of clusters generated by Eq. (1) to (3) with $\tau=0.1$ and $M_{\min }=0.08 M_{\odot}$. The thin solid curve is the KTG93 IMF normalized to the same total number of stars. All histograms are fractions of stars in each logarithmic mass interval of 0.05 dex

\section{Results}

Table 3 gives the parameters for the four main two-step IMF cases we have computed with the MC approach using Eqs. (1) to (3) and $\tau=0.10 . N_{\max }=10$ was chosen to represent the relatively wide cluster size distribution in Mot98. $N_{\text {ave }}(\approx 5)$ is the total number of stars $N_{\text {stars }}$ plus the total number of brown dwarfs $N_{\mathrm{bd}}$ divided by the total number of clusters (100000) in the ensemble. A fixed $N=5$ provides a good comparison with the variable $N$ cases. $M_{\min }=0.01 M_{\odot}$ and $0.08 M_{\odot}$ were used in order to explore differences between cases where brown dwarfs do and do not participate equally with stars in the pairing process (see also Sterzik \& Durisen 1999). Choosing $M_{\text {min }}=0.01 M_{\odot}$ yields about 20 to $25 \%$ brown dwarfs for the SMS in Eq. (3).

Table 3. Ensembles of 100000 clusters calculated with the MC approach

\begin{tabular}{lccccc}
\hline$N$-type & $N$ or $N_{\max }$ & $M_{\min }$ & $N_{\text {stars }}$ & $N_{\text {bd }}$ & $N_{\text {ave }}$ \\
\hline fixed & 5 & 0.08 & 500000 & 0 & 5 \\
& 5 & 0.01 & 394252 & 105748 & 5 \\
\hline variable & 10 & 0.08 & 480406 & 0 & 4.80 \\
& 10 & 0.01 & 441325 & 87830 & 5.30 \\
\hline
\end{tabular}

Table 4 shows the distribution of cluster sizes for $N_{\max }=10$. The first number in each entry is the number of clusters of size $N$; the second number is the average $M_{\text {tot }}$ for clusters of this size in the ensemble; and the third 
Table 4. Distribution of cluster sizes and average cluster masses for $N_{\max }=10$

\begin{tabular}{rrrrrrr}
\hline$N$ & \multicolumn{3}{c}{$M_{\min }=0.08$} & \multicolumn{3}{c}{$M_{\min }=0.01$} \\
\hline 1 & 6827 & 0.86 & 0.86 & 5117 & 0.92 & 0.92 \\
2 & 15846 & 0.88 & 0.44 & 11491 & 0.91 & 0.45 \\
3 & 17893 & 1.03 & 0.34 & 14772 & 1.05 & 0.35 \\
4 & 14124 & 1.40 & 0.35 & 13952 & 1.29 & 0.32 \\
5 & 10054 & 1.91 & 0.36 & 11556 & 1.68 & 0.36 \\
6 & 7685 & 2.56 & 0.44 & 9539 & 2.20 & 0.37 \\
7 & 6840 & 3.33 & 0.48 & 8533 & 2.88 & 0.41 \\
8 & 6494 & 4.00 & 0.50 & 8037 & 3.55 & 0.44 \\
9 & 6870 & 4.62 & 0.51 & 8139 & 4.13 & 0.46 \\
10 & 7367 & 5.29 & 0.53 & 8864 & 4.66 & 0.47 \\
\hline
\end{tabular}

entry is the average stellar mass for clusters of this size. The modal cluster size is only $N=3$ in both cases, but the cluster size distribution is somewhat more uniform with brown dwarfs. Both cluster size distributions tend to level off to a roughly constant value as $N \longrightarrow N_{\max }$. This means that imposition of an $N_{\max }$ is somewhat artificial, but $N_{\max }=10$ is roughly consistent with the range of apparent cluster sizes observed by Mot98. Note that the $f_{\mathrm{c}}\left(M_{\mathrm{tot}}\right)$ in Eq. (2) predicts an overall average cluster mass of $2.236 M_{\odot}$.

\subsection{Comparison of the resultant $f_{\mathrm{i}}(M)$}

Figure 2 shows the IMF's for the four cases in Table 3. Recall from Fig. 1 that parameters were optimized to give a decent $f_{i}(M)$ for the variable $N$ case without brown dwarfs. Use of a fixed $N=5$ degrades the agreement of the IMF with KTG93. The $f_{i}$ for fixed $N$ has too shallow a slope for moderate to high masses. For large $M_{\text {tot }}$, disallowing $N>5$ forces the creation of more high-mass stars at the expense of smaller masses. Introducing brown dwarfs does not degrade the variable $N$ IMF as seriously as imposition of a fixed $N$. The deviation consists of a slightly shallower slope at high masses. We do not attempt to fit any particular form of the IMF in the brown dwarf region, because this is still uncertain observationally (Marcy et al. 2000).

\subsection{Binary fractions}

Table 5 gives the overall binary fractions as a function of mass and spectral type along the main sequence for the four cases from Table 3. Tabulated are the number of MC binaries in the ensemble whose primaries lie in the stated mass range divided by the total of these binaries plus all single stars in the mass range. Thus a star in the mass range which is the secondary mass of a binary is not included in the $B F$ statistic. This is consistent with the way that observational results are presented in DM91, FM92, and L97. The fixed $N=5$ cases are denoted by

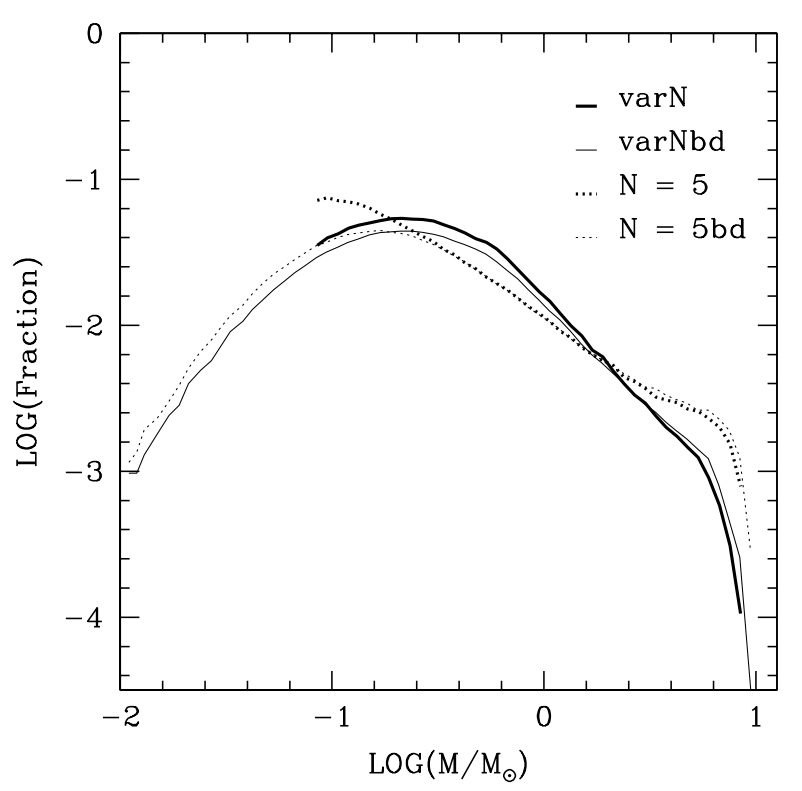

Fig. 2. The resultant two-step $f_{i}$ for the four cases in Table 3. The solid curves are for variable $N$; the dotted curves for fixed $N=5$. The heavy curves have no brown dwarfs; the lighter curves extending to lower masses are cases which include brown dwarfs. All histograms are fractions of stars or brown dwarfs in each logarithmic mass interval of 0.05 dex

a " 5 "; the variable $N$ cases by "var $N$ ". Cases run with brown dwarfs have the additional label "bd".

The mass boundaries in Table 5 are based on the one Gigayear isochrone of Yi et al. (2001) for solar composition. Allen (1976) is used to calibrate the conversion from effective temperature to spectral type. The boundary between $\mathrm{G}$ and $\geq \mathrm{F}$ is set a bit higher than the G0 mass of $1.14 M_{\odot}$ in $\mathrm{Yi}$ et al. because DM91 extend their statistics for solar-type stars into late $\mathrm{F}$ spectral types. The M stars are split near the mid-M's because there is a steep gradient of $B F$ in our results across the $M$ star mass range while the observational studies of $\mathrm{M}$ star binary frequency (FM92, L97) are biased towards early-M types.

Table 5. Binary fractions as a function of spectral class

\begin{tabular}{lccccc}
\hline sp-type & masses & 5 & $5 \mathrm{bd}$ & $\operatorname{var} N$ & $\operatorname{var} N \mathrm{bd}$ \\
\hline $\mathrm{L}$ & $<0.08$ & - & $2.2(-4)$ & - & $2.6(-4)$ \\
late M & $0.08-0.27$ & 0.11 & 0.12 & 0.04 & 0.05 \\
early M & $0.27-0.47$ & 0.35 & 0.45 & 0.31 & 0.35 \\
$\mathrm{~K}$ & $0.47-0.84$ & 0.54 & 0.61 & 0.48 & 0.51 \\
$\mathrm{G}$ & $0.84-1.20$ & 0.73 & 0.77 & 0.64 & 0.67 \\
$\geq \mathrm{F}$ & $>1.20$ & 0.92 & 0.93 & 0.86 & 0.87 \\
\hline
\end{tabular}

Figure 3 illustrates the $B F(M)$ 's for stars in more detail. The variable $N$ ensembles fit the $B F$ data on $\mathrm{M}$ and $\mathrm{G}$ stars rather well. The introduction of brown dwarfs to either fixed $N$ or variable $N$ cases tends to increase the 


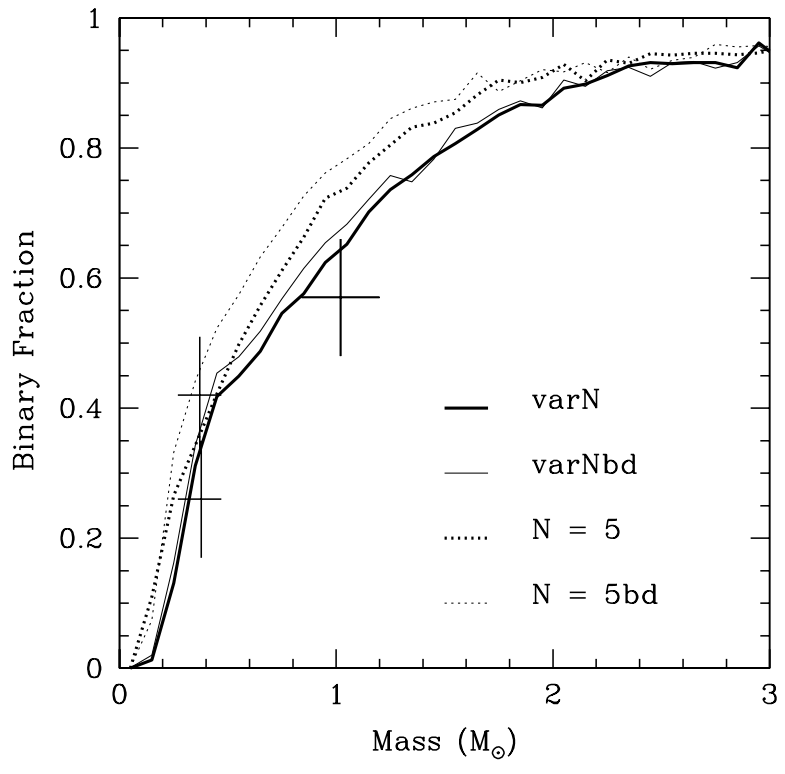

Fig. 3. The $B F(M)$ 's for the four cases in Tables 3 and 5 . The solid curves are for variable $N$; the dotted curves for fixed $N=$ 5 . The heavy curves have no brown dwarfs; the lighter curves are the cases which include brown dwarfs. For comparison, the data points from DM91, FM92, and L97 are plotted as crosshairs

binary fractions of the stars by amounts ranging from 10 's\% down to $\sim 1 \%$ as mass increases. This is simply because brown dwarfs are rarely binary primaries in our ensembles, and the stellar mass objects must take up the slack. The effect is larger for fixed $N$. In fact, the solartype $B F$ for the fixed $N$ case becomes uncomfortably large, especially with brown dwarfs. At first glance, the larger $B F$ for all masses in the fixed $N$ cases may seem inconsistent, because, with $N_{\text {ave }} \approx 5$ (see Table 3) for all ensembles, the total number of binaries should be similar. The systematically larger $B F(M)$ is possible, however, because the $f_{i}(M)$ are also significantly different between the fixed $N$ and variable $N$ cases (see Fig. 2). The total number of binaries remains 100000 in all cases, as required.

\subsection{Relative importance of incomplete biasing, a mix of cluster sizes, and the two-step process}

Our approach differs in several respects from McDC93. They used a one-step process with complete dynamical biasing and compared results for different fixed cluster sizes. For our variable $N$ cases, we consider a mix of cluster sizes together with incomplete biasing and two-step mass selection. To disentangle the relative importance of these effects for each case in Tables 3 and 5, we have used the identical sets of stars to re-select binaries in two ways: 1) We use the same clusters selected by the two-step process but assume only $M_{1} M_{2}$ binaries result (i.e., we use weights of 1.0, 0.0, and 0.0 for the mass pairings in Table 1). We refer to this as "complete" biasing statistics in Table 6. 2) We use the same stars and the same distribution of cluster sizes but randomize the star/cluster assignments and assign binaries by assuming complete dynamical biasing. This reproduces what McDC93 would obtain by choosing stars randomly from the same $f_{i}$ and creating the same distribution of cluster sizes, but without imposing a cluster total mass constraint.

Table 6. Comparison with binary fractions from complete biasing and a one-step IMF

\begin{tabular}{lccccc}
\hline sp-type & statistics & 5 & $5 b d$ & $\operatorname{var} N$ & $\operatorname{var} N b d$ \\
\hline early M & two-step & 0.35 & 0.45 & 0.31 & 0.35 \\
& complete & 0.35 & 0.46 & 0.32 & 0.35 \\
& McDC93 & 0.47 & 0.47 & 0.22 & 0.25 \\
\hline G & two-step & 0.73 & 0.77 & 0.64 & 0.67 \\
& complete & 0.79 & 0.84 & 0.66 & 0.70 \\
& McDC93 & 0.94 & 0.94 & 0.85 & 0.86 \\
\hline
\end{tabular}

Table 6 compares the $B F$ 's from the three approaches. We single out the early-M stars and G stars because these are the ones for which some observational data are published. A plot showing the effect on $B F(M)$ in more detail is given in Fig. 4 for the $\operatorname{var} N$ case. The effect of going from incomplete to complete biasing alone is an increase in the $B F$ 's of less than about $1-2 \%$ and $4-9 \%$ for early-M stars and $\mathrm{G}$ stars, respectively, typically within the uncertainties in the observed BF's. However, use of full McDC93 statistics results in dramatic changes. For $\operatorname{var} N$, it shifts the $B F$ for early-M stars downward and the $B F$ for $G$ stars upward by about $30 \%$ each! The agreement with observations is this case is seriously degraded, especially for the $\mathrm{G}$ stars. We conclude from Table 6 that the acceptable character of our two-step $B F(M)$ is due almost entirely to the imposition of the $M_{\text {tot }}$ constraint in the two-step process, not to the mixture of cluster sizes nor to the relaxation of "complete" biasing.

\subsection{The secondary mass distribution}

Another important characteristic of binary stars is the distribution of their mass ratios, $q=M_{\mathrm{s}} / M_{\mathrm{p}}$, where $M_{\mathrm{p}}$ is the mass of the primary and $M_{\mathrm{s}}$ is the mass of the secondary defined so that $M_{\mathrm{p}}$ is always $\geq M_{\mathrm{s}}$. The observed $q$-distributions for M stars in FM92 and L97 are flat near $q=1$ and drop smoothly to zero for mid- $q$ values. For G stars, DM91 indicate a strong tendency for the distribution to rise smoothly to a peak at about $q=0.25$. McDC93 have already shown that, for fixed $N$, choosing stellar masses from an IMF in a one-step process tends to produce such systematics in the $q$-distribution as a function of primary mass. As $M_{\mathrm{p}}$ increases, the $q$-distribution becomes more and more peaked toward low $q$. Figure 5, for the $\operatorname{var} N$ case from Tables 3 and 5, shows similar trends in the $q$-distributions for either our two-step or the McDC93 one-step approach. The shapes are perhaps marginally better in the two-step case, because the peak 


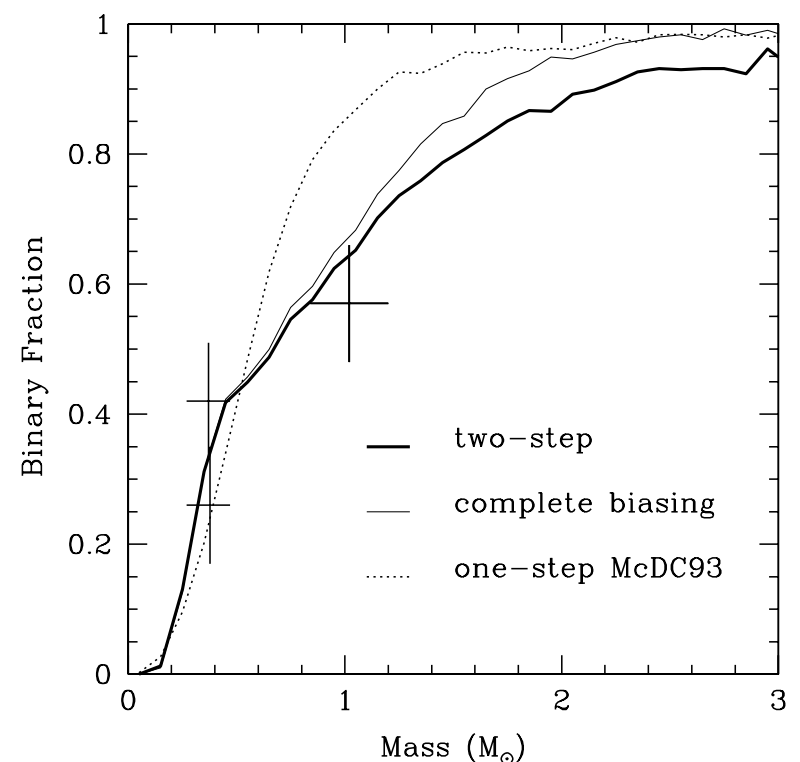

Fig. 4. A comparison of $B F(M)$ 's for the $\operatorname{var} N$ case using our "two-step" approach with incomplete biasing (heavy solid curve) and with "complete" biasing (thin solid curve). The dotted curve "McDC93" is obtained from the same cluster size and stellar mass distributions by choosing stars in a one-step process without an $M_{\text {tot }}$ constraint using complete biasing. The cross-hairs have the same meaning as in Fig. 3

in the $\mathrm{G}$ star distribution shifts to lower $q$ compared to McDC93.

As illustrated in Fig. 6, the introduction of brown dwarfs and a fixed cluster size, both tend to make the G star $q$-distribution more peaked at low $q$. Unfortunately, as shown in Table 5, this comes at the cost of making the $\mathrm{G}$ star $B F$ higher and hence less acceptable.

\subsection{Varying input parameters}

In this subsection, we report how changes in each major parameter affects the quality of results when they are varied one at a time about the values used for the $\operatorname{var} N$ calculation. For reference, the standard $\operatorname{var} N$ value of the parameter is given in parentheses at the start of each paragraph.

Tolerance $\tau(0.10)$. The low and variable efficiency of gas utilization by star formation makes it difficult to know what value of $\tau$ to use. Increasing $\tau$ from 0.1 to 0.25 weakens the $M_{\text {tot }}$ constraint, and distributions tend to shift somewhat toward one-step results. The $f_{i}$ looks more like $f_{\mathrm{s}}$ and has a distinct deficit of stars above about $5 M_{\odot}$ compared with the KTG93 IMF. The BF's for early-M, $\mathrm{K}$, and $\mathrm{G}$ stars all increase by about 6 or $7 \%$. When $\tau$ is decreased from 0.10 to 0.05 , the IMF is slightly closer to KTG93, especially near a solar mass. Although the $B F$ 's do not change to within a percent when $\tau$ is reduced, the $q$-distribution for $\mathrm{G}$ stars becomes slightly closer to the DM91 distribution.

Maximum Cluster Size $N_{\max }$ (10). Changing $N_{\max }$ from 10 to 15 has a similar but greater effect on $f_{i}$ as an in-

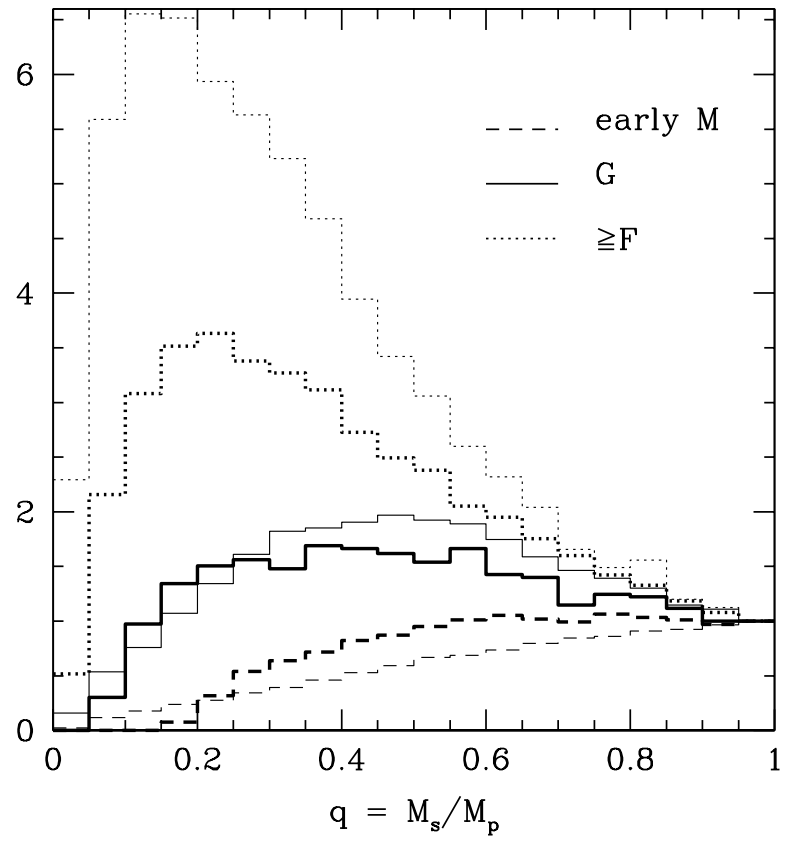

Fig. 5. Histograms of $q$-distributions for the var $N$ case. Three spectral class bins from Table 5 are shown, early M, G, and $\geq$ F. Distributions are all normalized to unity for the $q=0.95$ to 1.00 bin in order to emphasize comparison of the distribution shapes. The heavy curves are for the two-step process; the thin curves are for the equivalent McDC93 method of selecting cluster members

crease in $\tau$. It effectively weakens the $M_{\text {tot }}$ constraint and causes $f_{i} \rightarrow f_{\mathrm{s}}$. For $N_{\max }=15$, there is a severe deficit of stars more massive than a few $M_{\odot}$ compared to KTG93. On the other hand, when $N_{\max }$ is decreased to 7 , the $M_{\text {tot }}$ constraint strengthens to produce a substantial excess of high-mass stars over KTG93. Although the G star BF fluctuates nonmonotonically by a few percent as $N_{\max }$ increases from 7 to 15 , the $B F$ of early-M stars systematically decreases from 0.38 to 0.27 . The $q$-distribution of the G stars looks more like the DM91 distribution as $N_{\max }$ increases.

CMS Power-Law Index $\alpha_{\mathrm{c}}$ (1.5). As $\alpha_{\mathrm{c}}$ increases from 1.1 to $2.0, f_{i}$ goes from a severe overabundance to a severe deficit relative to KTG93 for stars of a solar mass or above, while $B F$ 's increase systematically. The early-M star $B F$ increases from 0.20 to 0.48 , while the $\mathrm{G}$ star $B F$ rises more moderately from 0.57 to 0.70 . The resemblence of the G star $q$-distribution to DM91 improves as $\alpha_{\mathrm{c}}$ goes up.

Upper Mass Limit for $M_{\text {tot }}\left(10 M_{\odot}\right)$. Everything is degraded by decreasing the upper limit for $M_{\text {tot }}$ from 10 to $5 M_{\odot}$. The $B F$ for $\mathrm{G}$ stars climbs to 0.83 , the IMF is a very poor match to KTG93, and the G star $q$-distribution has a broad, low peak near $q=0.4$. When the upper limit for $M_{\text {tot }}$ is increased from 10 to $15 M_{\odot}$, the $M_{\text {tot }}$ constraint forces a large excess of massive stars over KTG93, and the $B F$ 's for early-M, K, and G stars drop by 8 to $11 \%$. The $q$ distribution for the $\mathrm{G}$ stars becomes a poorer fit to DM91.

Upper Mass Limit for the Stars $\left(10 M_{\odot}\right)$. In Sect. 2.3, the upper mass limits for the clusters and stars are taken 


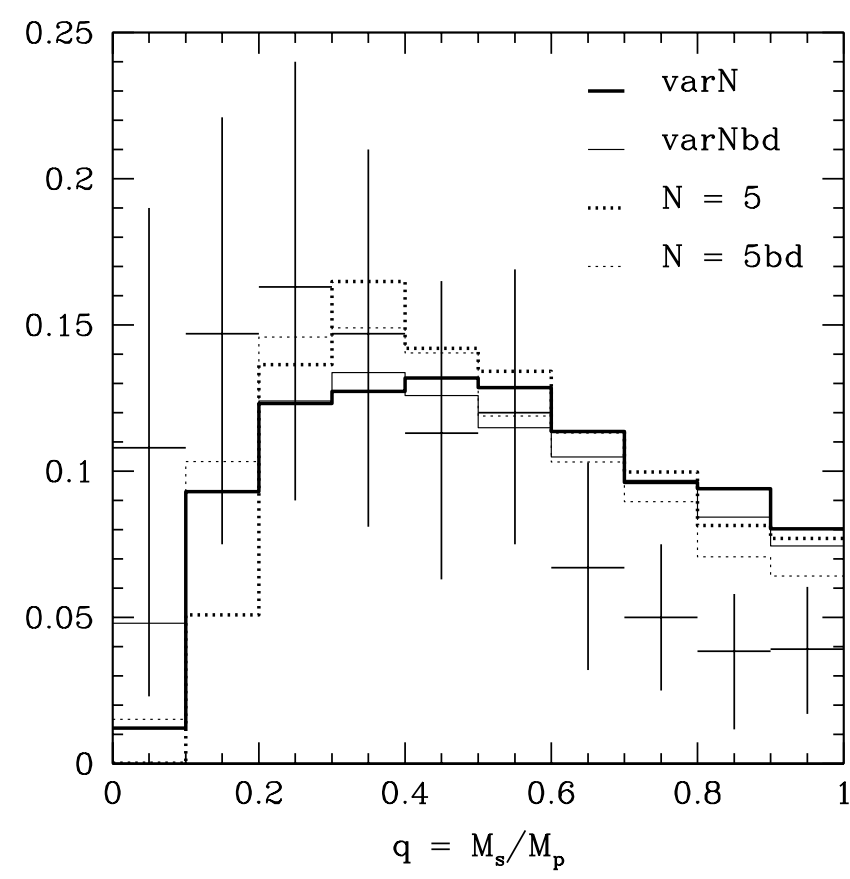

Fig. 6. Histograms of the G star $q$-distribution for the four cases in Tables 3 and 5 . The solid lines are for variable $N$; the dotted lines for fixed $N=5$. The heavy lines have no brown dwarfs; the lighter lines are the cases which include brown dwarfs. For comparison, the data from DM91 are plotted as cross-hairs. All distributions are normalized to the same total number of binaries as for $\operatorname{var} N$

to be the same. If the stellar upper mass limit is reduced from 10 to $5 M_{\odot}$, there is little impact on any results, except that there is now a larger excess of stars between 2 and $5 M_{\odot}$ over KTG93. Changes in the $B F$ 's are only at the 1 to $3 \%$ level.

SMS Peak $\mu\left(\log _{10} 0.20\right)$. The parameter $\mu$ is defined so that it represents the peak, in solar units, that the lognormal $f_{\mathrm{s}}$ has when plotted in a $\log M$ histogram. In fact, for our standard choice of all other parameters, the $f_{i}$ exhibits essentially this same peak. For $\mu=\log _{10} 0.30$, the relatively strong resulting deficit of low-mass stars is compensated by a large excess in higher-mass stars. The IMF is a rather poor match to KTG93. For $\mu=\log _{10} 0.15$, $f_{i}$ stays closer (within about $20 \%$ ) to the KTG93 over a much wider range of masses. The $\mu=\log _{10} 0.30$ case tends to drive the early-M, K, and G star $B F$ 's down by roughly $10 \%$, while $\mu=\log _{10} 0.15$ increases them by 5 to $7 \%$. The G star $q$-distribution is more like DM91 for $\mu=\log _{10} 0.15$.

SMS Width $\sigma\left(\log _{10} 0.30\right)$. Setting $\sigma$ to $\log _{10} 0.25$ effectively makes the lognormal distribution in (3) broader and produces an excess of high-mass stars over KTG93. The $\mathrm{M}$ star and $\mathrm{G}$ star $B F$ 's go up and down, respectively, by a few percent each; and the $\mathrm{G}$ star $q$-distribution becomes somewhat more like DM91. A $\sigma$ change in the other direction, to $\log _{10} 0.40$, which narrows the distribution, has quantitatively similar effects in the opposite direction, except that the M star BF is essentially the same; and the IMF does not differ as much from KTG93.
Overall, there is a tendency for any change which produces fewer high-mass stars in the IMF to increase the $B F$ for $\mathrm{G}$ stars while making their $q$-distribution look more like the DM91 distribution. One interesting exception is a decrease in $\tau$. As the $M_{\text {tot }}$ constraint is strengthened by decreasing $\tau$ below 0.10 , the IMF and G star $B F$ remain essentially the same, while the $q$-distribution improves slightly. In effect, the degree of dynamical biasing remains nearly the same, while the two-step nature of the mass selection is enhanced. The tolerance $\tau$ seems unique in this respect.

In a preliminary report on this work, Durisen et al. (2000) presented results for a "best case" choice of parameters. We have made a similar effort here by making several minor tweaks to the $\operatorname{var} N$ parameters as follows: $\tau=0.05, N_{\max }=12, \alpha_{\mathrm{c}}=1.7$, and $\mu=\log _{10} 0.18$. The upper mass cutoff for the CMS was increased to $15 M_{\odot}$, and the upper mass limit for the SMS was reduced to $7.5 M_{\odot}$. We put "best case" in quotes to indicate there has been no systematic optimization. The IMF and $q$ distributions are not markedly changed; but, as shown in Fig. 7 , the observations of $B F(M)$ can be matched well by these minor changes.

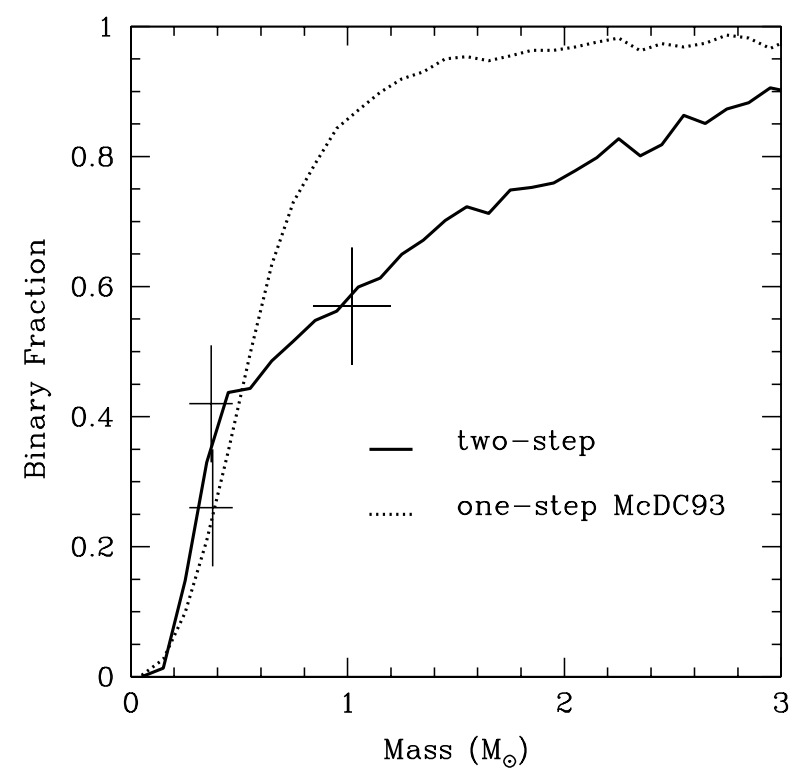

Fig. 7. The $B F(M)$ for the "best case" using our two-step approach with incomplete biasing (heavy solid curve). The dotted curve "McDC93" is obtained from the same cluster size and stellar mass distributions by choosing stars in a one-step process without an $M_{\text {tot }}$ constraint. The cross-hairs have the same meaning as in Fig. 3

\subsection{Effects of the $f_{\mathrm{c}}\left(M_{\text {tot }}\right)$ and $f_{\mathrm{s}}(M)$ functional forms}

We test the sensitivity of our two-step IMF results to the shapes chosen for the CMS and SMS by computing three additional ensembles. We refer to them by names which designate first the shape of the CMS and then the shape of the SMS. In this notation, the $\operatorname{var} N$ ensemble becomes 


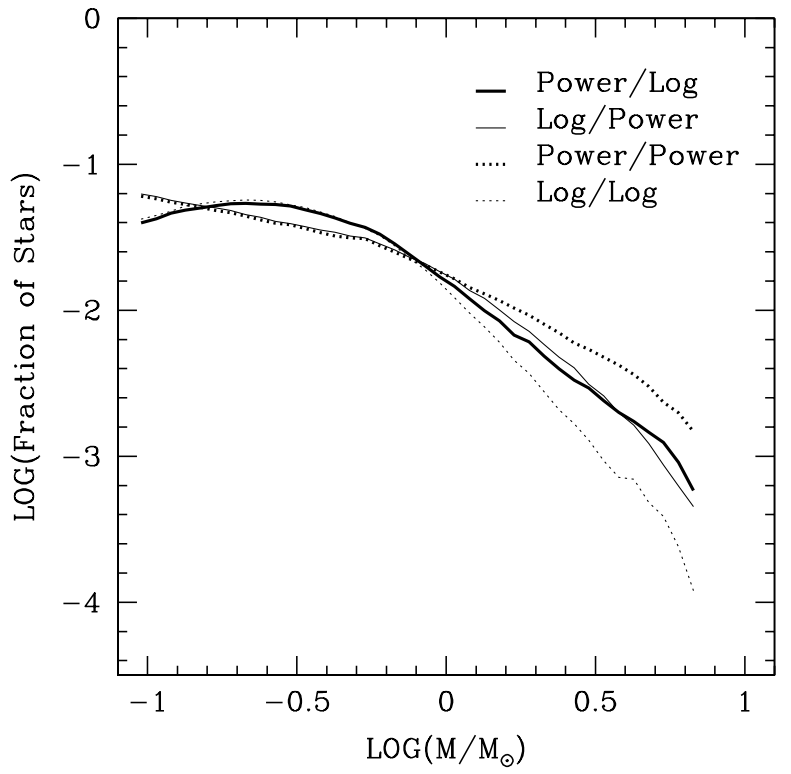

Fig. 8. The resultant two-step $f_{i}$ for the different choices of CMS and SMS in Table 7. The heavy solid line is $\operatorname{var} N$, the standard power-law CMS with a lognormal SMS. The thin solid curve is LogPow; the heavy dotted, PowPow; the light dotted LogLog. All histograms are fractions of stars in each logarithmic mass interval of 0.05 dex

"Power/Log", meaning that the CMS is a power law and the SMS a lognormal. These designations are further abbreviated in obvious ways as needed. For the new ensembles with variable $N$, the $\tau$, the $N_{\max }$, and the mass limits of the distributions are kept the same as for the var $N$ case; and all power laws have the same index $\alpha=1.5$. When a lognormal is used for the SMS, it is identical to (3). However, this distribution peaks at too low a mass and is too narrow for a proper CMS. So, when a lognormal is used for the CMS, we choose $\sigma=\log _{10} 0.38$ and $\mu=\log _{10} 0.90$. These values produce a reasonable fit to the lognormal obtained by Klessen et al. (1998) from simulations of gravitational interactions and turbulence in a molecular cloud, after it is scaled to give a peak cluster mass at $0.9 M_{\odot}$.

Figure 8 compares the two-step IMF's from the four ensembles. For reference, remember, from Fig. 1, that the standard Power/Log case does a reasonably good job of reproducing the KTG93 IMF. The Log/Power IMF actually fits the piecewise power-law KTG93 better at low masses but is slightly worse than Power/Log at the highmass end. The Log/Log and Power/Power cases exhibit stronger, possibly unacceptable deviations. Power/Power fits KTG93 well enough at low and moderate masses, but it severely overproduces stars more massive than about $2 M_{\odot}$. The $\log / \log$ case is very similar to Power $/ \mathrm{Log}$ at low and moderate masses, but underproduces massive stars. It might be possible to improve any one of these cases by varying parameters. We have not done so here, because there is little justification yet for any particular choices.

Despite the range of mass spectra, the two-step mass selection results in a similar $B F$ pattern for these cases,
Table 7. Binary fractions as a function of spectral class for different CMS and SMS choices

\begin{tabular}{lcccc}
\hline sp-type & PowLog & LogPow & PowPow & LogLog \\
\hline late M & 0.04 & 0.04 & 0.04 & 0.03 \\
early M & 0.31 & 0.48 & 0.41 & 0.38 \\
K & 0.48 & 0.66 & 0.54 & 0.63 \\
G & 0.64 & 0.75 & 0.65 & 0.77 \\
$\geq$ F & 0.86 & 0.81 & 0.81 & 0.88 \\
\hline
\end{tabular}

as shown in Table 7 . The range of BF's for each spectral type in Table 7 is similar to the range in Table 5 . Use of a lognormal for the CMS does about as much damage to the $\mathrm{G}$ star $B F$ as imposition of a fixed $N=5$. Tests of these ensembles for the effects of complete biasing and the use of one-step McDC93 statistics yield results similar to those in Fig. 4. The form of $B F(M)$ is strongly influenced by the $M_{\text {tot }}$ constraint in the two-step mass selection. Compared with McDC93, two-step mass selection tends to increase the $\mathrm{M}$ star $B F$ and decrease the $\mathrm{G}$ star $B F$.

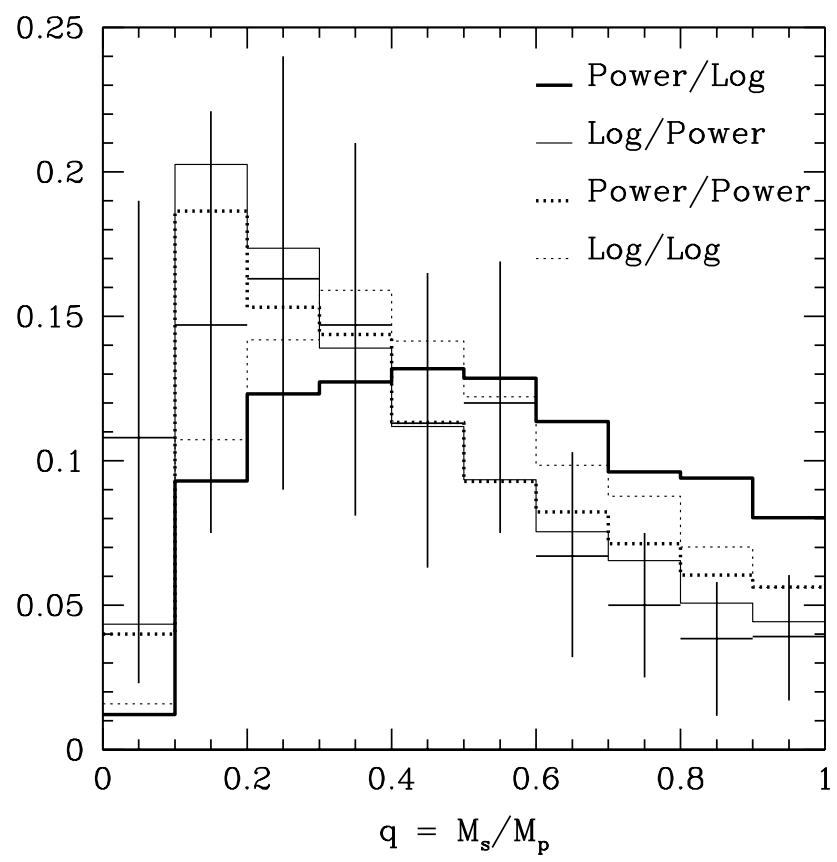

Fig. 9. Histograms of the $\mathrm{G}$ star $q$-distribution for the four cases in Table 7 . The heavy solid line is $\operatorname{var} N$, the standard power-law CMS with a lognormal SMS. The thin solid curve is LogPow; the heavy dashed, PowPow; the heavy dotted LogLog. For comparison, the data from DM91 are plotted as cross-hairs. For comparison purposes, all distributions are normalized to the same total number of binaries as for $\operatorname{var} N$

Figure 9 illustrates the most important qualitative as well as quantitative change that results from varying the shapes of the mass spectra. All the cases other than Power/Log have G star $q$-distributions that are much more like the DM91 data. In the Log/Log and Log/Power cases, this comes at the expense of a $\mathrm{G}$ star $B F$ of $\sim 0.76$ which 
borders on unacceptable; but, in the Power/Power case, the $B F$ 's are as good as they are for the var $N$ Power $/ \mathrm{Log}$ standard. On the other hand, the Power/Power ensemble has far too many high-mass stars in its IMF. There is no one case we have identified which simultaneously satisfies all criteria for "goodness", but the Power/Power case does demonstrate that good $B F$ 's and a good G star $q$ distribution can be obtained at the same time, something which was not evident in Sect. 3.5, when we restricted ourselves to Power/Log.

\section{Discussion}

It is perhaps important now to reiterate that we consider all computations in this Paper to be illustrative, not definitive. There are two fundamental reasons: a) the proper choices of input parameters and mass spectra are not yet known and b) our analysis implicitly assumes that most stars experience a few-body cluster decay governed primarily by point-mass dynamics. We can be assured that assumption (b) is wrong because the early dynamics of young stellar few-body systems must be affected by ongoing gas accretion (Bonnell et al. 1997, 1998) and possibly also by dissipative interactions with and between circumstellar disks (McDC95). In fact, a good deal of gas is present in observed young multiples that seem to be undergoing decay (Reipurth 2000).

What we do show, however, is that, if a large fraction of stars form in small clusters, the imprint of a cluster total mass constraint through a two-step mass selection can, by itself, have a profound effect on mass-dependent properties of binary statistics. The difference in $B F$ between one-step and two-step approaches is in fact larger near $M=M_{\odot}$ than that reported in McDC95 between one-step calculations which include strong disk collisions and those which do not. Imposition of our CMS $M_{\text {tot }}$ constraint thus produces larger desirable shifts in the BF's than the introduction of strongly dissipative interactions in the cluster decay dynamics! Future, more complete observational determinations of $B F(M)$ and $q$-distributions are on the horizon (see reviews in Zinnecker \& Mathieu 2001) and should provide important diagnostics for binary formation mechanisms and contributing processes.

A particular difficulty with few-body decay as a binary production mechanism is that brown dwarf binaries apparently exist (Basri 2001). Even with a two-step IMF, dynamical biasing causes the lightest masses in the final $f_{i}$ to be preferentially single. If few-body decay is an important mechanism for star formation, then finding brown dwarf binaries to be common would suggest that brown dwarfs do not participate in the same few-body dynamics as young stars but must be formed by a separate mechanism. A possible alternative might be that the lower mass limit on the CMS effectively extends down to rather low masses. On the other hand, the $B F$ of brown dwarfs is completely unknown. The so-called "brown dwarf desert", i.e., the minimum in the companion mass function between the masses of low-mass stars and planets in ra- dial velocity surveys (Halbwachs et al. 2000; Marcy et al. 2000), appears to be statistically significant and might well be an imprint of dynamical biasing (McDC93). At the same time, estimates for the population of apparently freefloating brown dwarfs in the field and in young clusters is growing dramatically with the advent of large-scale, highly sensitive photometric surveys (e.g., Reid 1999; Meyer et al. 2000).

\section{Conclusions}

When the constraint of selecting the total mass from a cluster (or cloud core) mass spectrum is included in the statistics of choosing stellar masses for cluster members, the decay of few-body star clusters yields binary fractions as a function of spectral type which agree reasonably well with published observations, while still producing an acceptable stellar IMF. In fact, the improvement in $B F$ 's is greater than what can be achieved by the inclusion of strongly dissipative interactions in the dynamics of the few-body system. Although proper choices for various input parameters and functional forms are still poorly known, we have shown that plausible assumptions can give acceptable results for the IMF, $B F(M)$, and the secondary mass distribution of $\mathrm{G}$ star binaries. Admittedly, it is somewhat difficult to obtain good results for all three simultaneously. Our primary point is that the predictions for binary characteristics from few-body cluster decay are strongly affected, in a generally favorable direction, by including a cluster total mass constraint in the statistics. Few-body decay with two-step mass selection is therefore worthy of further consideration as a potentially important component of the star formation process.

Acknowledgements. We would like to thank S. Aarseth, J. Alcalá, M. R. Bate, I. Bonnell, A. Burkert, C. Clarke, C. Deliyannis, B. G. Elmegreen, T. Hartquist, L. Kiseleva, R. Klein, P. Kroupa, R. Larson, E. Levy, C. McKee, R. Neuhäuser, B. Reipurth, and H. Zinnecker for useful conversations and encouragement on aspects of this research over the years. We especially thank the referee F. Palla, whose helpful comments made this a substantially more readable paper. This work was supported in part by NASA Grants NAGW 3399 and NAGW54342. Most of the research was done while R.H.D. was an Alexander von Humboldt Awardee at Max Planck Institute for Extraterrestrial Physics in Munich. B.K.P.'s efforts were supported in part by a NASA-ASEE Summer Faculty Fellowship.

\section{References}

Adams, F. C., \& Fatuzzo, M. 1996, ApJ, 464, 256

Allen, C. W. 1976, Astrophysical Quantities (Athlone Press: London)

Basri, G. 2001, in The Formation of Binary Stars, ed. H. Zinnecker, \& R. D. Mathieu (ASP: San Francisco)

Bate, M. R. 1999, MNRAS, 314, 33

Bonnell, I. A., Bate, M. R., Clarke, C. J., \& Pringle, J. E. 1997, MNRAS, 285, 201

Bonnell, I. A., Bate, M. R., \& Zinnecker, H. 1998, MNRAS, 298, 93 
Burkert, A., \& Bodenheimer, P. 1996, MNRAS, 280, 1190

Clarke, C. J., \& Pringle, J. E. 1991, MNRAS, 249, 588

Clarke, C.J., \& Pringle, J. E. 1993, MNRAS, 261, 190

Duquennoy, A., \& Mayor, M. 1991, A\&A, 248, 485 (DM91)

Durisen, R. H., Pickett, B. K., \& Sterzik, M. F. 2000, in IAU Symp. 200, Poster Proceedings, ed. B. Reipurth, \& H. Zinnecker (Potsdam), 202

Elmegreen, B. G. 1997, ApJ, 486, 944

Elmegreen, B. G., \& Falgarone, E. 1996, ApJ, 471, 816

Fischer, D. A., \& Marcy, G. W. 1992, ApJ, 396, 178 (FM92)

Halbwachs, J. L., Arenou, F., Mayor, M., Udry, S., \& Queloz, D. $2000, \mathrm{~A} \& \mathrm{~A}, 355,581$

Harrington, R. S. 1974, Cel. Mech., 9, 465

Harrington, R. S. 1975, AJ, 80, 1081

Heggie, D. C. 1975, MNRAS, 173, 729

Klessen, R. S., Burkert, A., \& Bate, M. R. 1998, ApJ, 501, L205

Kroupa, P. 1998, in Brown Dwarfs and Extrasolar Planets, ed. R. Rebolo, E. L. Martin, \& M. R. Z. Osorio (ASP: San Francisco), 483

Kroupa, P., Tout, C. A., \& Gilmore, G. 1993, MNRAS, 262, 545 (KTG93)

Lada, E. A., DePoy, D. L., Evans, N. J., \& Gatley, I. 1991, ApJ, 371, 171

Larson, R. B. 1992, MNRAS, 256, 641

Leinert, C., Henry, T., Glindemann, A., \& McCarthy, D. W. 1997, A\&A, 325, 159 (L97)

Luhman, K. L., \& Rieke, G. H. 1999, ApJ, 525, 440

Marcy, G. W., Cochran, W. D., \& Mayor, M. 2000, in Protostars and Planets IV, ed. V. Mannings, A. P. Boss, \& S. S. Russell (U. Arizona Press: Tucson), 1285

McDonald, J. M., \& Clarke, C. J. 1993, MNRAS, 262, 800 (McDC93)

McDonald, J. M., \& Clarke, C. J. 1995, MNRAS, 275, 671 (McDC95)
Meyer, M. R., Adams, F. C., Hillenbrand, J. M., Carpenter, J. M., \& Larson, R. B. 2000, in Protostars and Planets IV, ed. V. Mannings, A. P. Boss, \& S. S. Russell (U. Arizona Press: Tucson), 121

Mikkola, S., \& Aarseth, S. J. 1990, Cel. Mech. Dyn. Astr., 47, 375

Mikkola, S., \& Aarseth, S. J. 1993, Cel. Mech. Dyn. Astr., 57, 439

Miller, G. E., \& Scalo, J. M. 1979, ApJS, 41, 513

Monaghan, J. J. 1976a, MNRAS, 176, 63

Monaghan, J. J. 1976b, MNRAS, 177, 583

Motte, F., Andre, P., \& Neri, R. 1998, A\&A, 336, 150 (Mot98)

Reid, I. N. 1999, in Star Formation 1999, ed. T. Nakamoto, Nagoya, 327

Reipurth, B. 2000, AJ, 120, 3177

Saslaw, W. C., Valtonen, M. J., \& Aarseth, S. J. 1974, ApJ, 190,253

Scalo, J. 1986, Fund. Cosmic Phys., 11, 1

Scalo, J. 1998, in The Stellar Initial Mass Function, ed. G. Gilmore, \& D. Howell (APS: San Francisco), 201

Smith, K. W., Bonnell, I. A., \& Bate, M. R. 1997, MNRAS, 288,1041

Standish, E. M. 1972, A\&A, 21, 185

Sterzik, M. F., \& Durisen, R. H. 1995, A\&A, 304, L9 (SD95)

Sterzik, M. F., \& Durisen, R. H. 1998, A\&A, 339, 95 (SD98)

Sterzik, M. F., \& Durisen, R. H. 1999, in Star Formation 1999, ed. T. Nakamoto, Nagoya, 387

Testi, L., \& Sargent, A. 1998, ApJ, 508, L91

Valtonen, M. J. 1976, Ap\&SS, 42, 331

van Albada, T. S. 1968a, Bull. Astron. Inst. Neth., 19, 479

van Albada, T. S. 1968b, Bull. Astron. Inst. Neth., 20, 57

Yi, S., Demarque, P., Kim, Y.-C. et al. 2001, ApJS, in press

Zinnecker, H. 1982, Ann. NY Acad. Sci., 395, 226

Zinnecker, H., \& Mathieu, R. D. 2001, The Formation of Binary Stars (ASP: San Francisco) 